\title{
TITLE:
}

\section{Dynamics of Large-Scale Eddies in Turbulent Flows}

$\operatorname{AUTHOR}(\mathrm{S})$ :

TATSUMI, Tomomasa

CITATION:

TATSUMI, Tomomasa. Dynamics of Large-Scale Eddies in Turbulent Flows. 数理解析研究所講究録 1986, 601: 217-227

ISSUE DATE:

1986-12

URL:

http://hdl.handle.net/2433/99607

RIGHT: 
Dynamics of Large-Scale Eddies in Turbulent Flows

\author{
By Tomomasa TATSUMI (要年正) \\ Hiroshima Institute of Technology (広島工業大学)
}

A model equation based on the equipartition of the turbulent dissipation is proposed for describing the dynamics of largescale eddies in turbulent flows. The equation is reducible to the equation of motion of an inviscid fluid, so that the motion of the large-scale eddies can be described in terms of inviscid fluid dynamics.

\title{
1. Introduction
}

A prominent feature of homogeneous turbulence at large Reynolds numbers is the existence of a dynamical equilibrium state

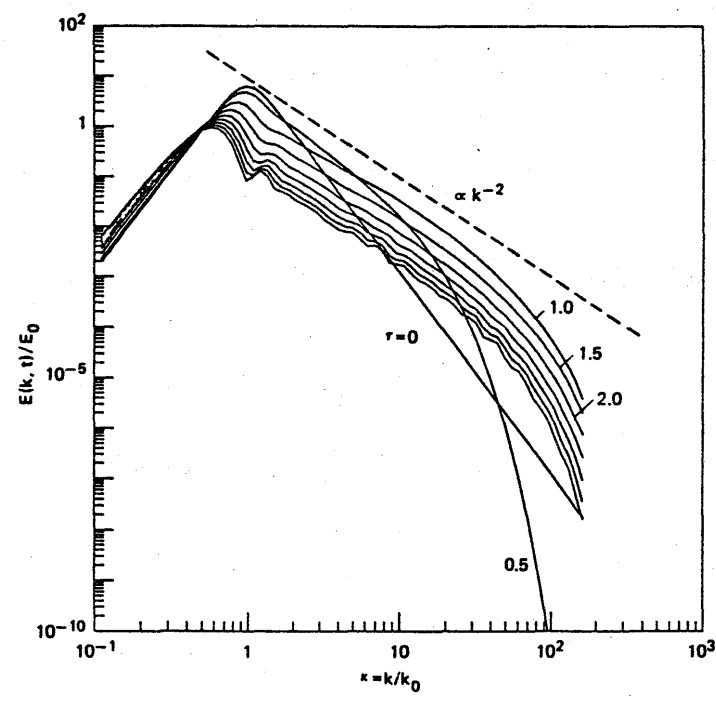

Fig. 1

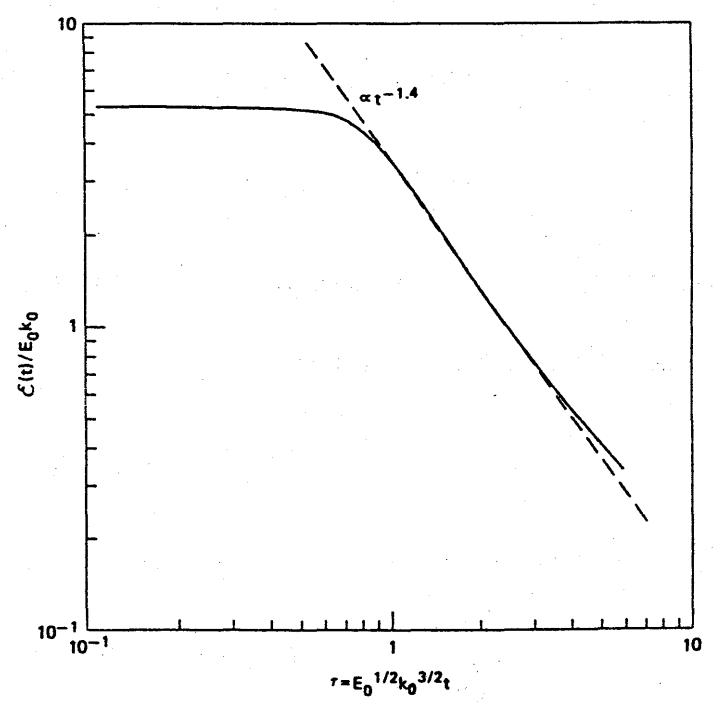

Fig. 2 
which is characterized by the self-similarity of statistical quantities during their evolution in time.

Figures 1 and 2 depict respectively the evolution of the energy spectrum $E(k, t)$ and the energy of turbulence,

$$
\varepsilon(t)=\frac{1}{2}\left\langle|u(x, t)|^{2}\right\rangle=\int_{0}^{\infty} E(k, t) d k,
$$

where $u(k, t)$ is the fluid velocity and the angular brackets denote the statistical average. These figures have been taken from the numerical result by Tatsumi et al. (1986) based on the factorized fourth cumulant approximation, but similar results are also provided by other works using different closure hypotheses (see Orszag (1977) and Tatsumi (1980)). The figures clearly show that the dynamical equilibrium state is attained about at the time $\tau=E_{0}{ }^{1 / 2} \mathrm{k}_{0}{ }^{3 / 2} t=0.8, E_{0}$ and $\mathrm{k}_{0}$ being the characteristic magnitudes of the energy spectrum and the wavenumber respectively. The numerical value of the critical time is common to all statistical quantities for a realization of turbulence but changeable according to the initial condition. The energy spectrum is governed by the equation,

$$
\left(\frac{\partial}{\partial t}+2 \nu k^{2}\right) E(k, t)=T(k, t) \text {, }
$$

where $T(k, t)$ denotes the energy transfer function which satisfies the condition,

$$
\int_{0}^{\infty} \mathrm{T}(\mathrm{k}, t) \mathrm{dk}=0 .
$$

The equation (1.2) may be rewritten as

$$
\left(\frac{\partial}{\partial t}+2 \gamma(k, t)\right) E(k, t)=0
$$

where 


$$
\gamma(k, t)=\nu k^{2}-T(k, t) / 2 E(k, t)
$$

represents the turbulent dissipation rate that is the net effect of the viscous dissipation and the nonlinear energy transfer in the wavenumber space.

Generally speaking, the turbulent dissipation rate $\gamma(k, t)$ is dependent on the wavenumber $k$, but if the energy spectrum satisfies a similarity law with respect to $k, \gamma(k, t)$ becomes independent of $\mathrm{k}$,

$$
\gamma(k, t)=\gamma_{0}(t) \text {. }
$$

Such an equipartition of the dissipation may clearly be observed in Fig. 3 which has been taken from the numerical result by Tatsumi et al. (1986). Initially, $\gamma(k, t)$ is proportional to $\mathrm{k}^{2}$ according to the initial condition $T(k, 0)=0$, but soon it develops a $k$-independent form for almost two decades of the wavenumber roughly corresponding to the energy-containing and the inertial ranges. The functional form of $\gamma(k, t)$ beyond the inertial range is not apparent on Fig. 3, but its asymptotic behaviour for very large $k$ is known to be $\gamma(k, t) \propto k$ (Tatsumi $(1980))$.

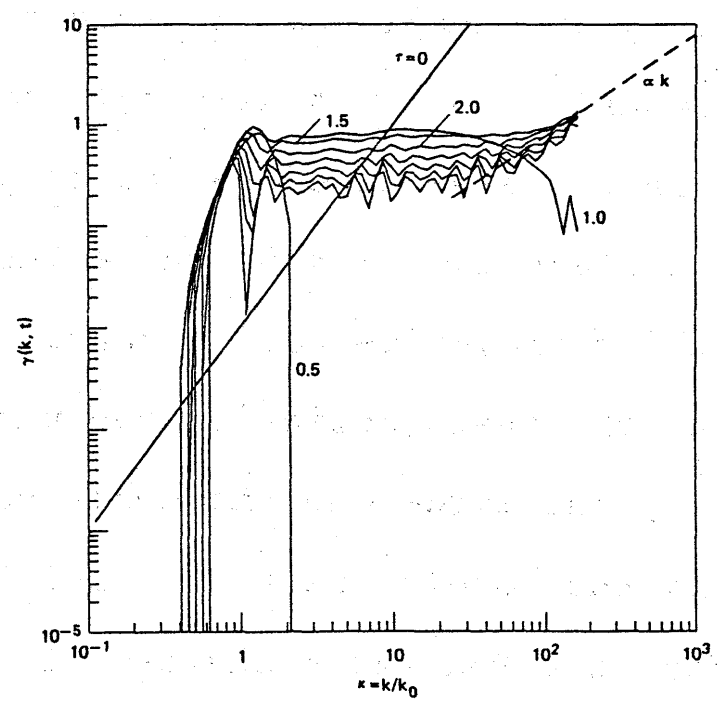

Fig. 3 
If we employ the equipartition form of the dissipation (1.6), the equation (1.4) is written as

$$
\left(\frac{\partial}{\partial t}+2 \gamma_{0}(t)\right) E(k, t)=0,
$$

which leads to the energy equation

$$
\varepsilon(t)=2 \gamma_{0} \varepsilon(t) \text {, }
$$

where

$$
\varepsilon(t)=-d \varepsilon(t) / d t
$$

denotes the viscous dissipation of the energy. Hence,

$$
\gamma_{0}(t)=\varepsilon(t) / 2 \varepsilon(t),
$$

which gives the expression of $\gamma_{0}(t)$ in terms of measurable quantities.

\section{Dynamics of large-scale eddies}

The balance of energy in homogeneous turbulence at large Reynolds numbers is conveniently dealt with by dividing the turbulent motion into large and small-scale components which are usually called "eddies". The large-scale eddies contain almost all kinetic energy of turbulence and lose their energy through the nonlinear transfer to the small-scale eddies. In the wavenumber space, the large-scale eddies correspond to the energycontaining range where $T(k, t)<0$, while the small-scale eddies correspond to the energy-dissipation range $T(k, t)>0$. If we denote by $k=k_{1}$ the characteristic wavenumber of the inertial range $T(k, t) \simeq 0$, the large and the small-scale eddies may be characterized by the conditions $\mathrm{k}<\mathrm{k}_{1}$ and $\mathrm{k}>\mathrm{k}_{1}$ respectively. If we define the Fourier transform of the fluid velocity 
$u(x, t)$ by

$$
u(x, t)=\int v(k, t) \exp (i k \cdot x) d k,
$$

where $v(k, t)$ should be understood as a generalized function, and express the velocity $u(x, t)$ as

$$
u(x, t)=\tilde{u}(x, t)+\hat{u}(x, t),
$$

where $\tilde{u}(x, t)$ and $\hat{u}(x, t)$ denote contributions from the large and the small-scale eddies respectively, these component velocities are expressed as

$$
\left.\begin{array}{l}
\tilde{u}(x, t)=\int_{|k|<k_{1}} v(k, t) \exp (i k \cdot x) d k, \\
\hat{u}(x, t)=\int_{|k|>k_{1}} v(k, t) \exp (i k \cdot x) d k .
\end{array}\right\}
$$

In the physical space, such a decomposition can be made by taking a moving time-average with a period which is larger than the characteristic time of the small-scale eddies but shorter than that of the large-scale eddies:

$$
\tilde{u}(x, t)=\langle u(x, t)\rangle_{e^{\prime}}\langle\hat{u}(x, t)\rangle_{e}=0,
$$

where the brackets \langle\rangle$_{e}$ denote the above-mentioned time-average. If we substitute (2.2) and the corresponding decomposition of the pressure,

$$
p(x, t)=\tilde{p}(x, t)+\hat{p}(x, t),
$$

into the equations of motion,

$$
\begin{aligned}
\frac{\partial u}{\partial t}+(u \cdot g r a d) u & =-\frac{1}{\rho} \operatorname{grad} p+v \Delta u, \\
\operatorname{div} u & =0,
\end{aligned}
$$

and take the average \langle\rangle$_{e}$, we obtain the following equations for the large-scale eddies: 


$$
\begin{aligned}
\frac{\partial \tilde{u}}{\partial t}+(\tilde{u} \cdot \operatorname{grad}) \tilde{u} & =-\frac{1}{\rho} \operatorname{grad} \tilde{p}+\left(\nu \Delta \tilde{u}-\langle(\hat{u} \cdot \operatorname{grad}) \hat{u}\rangle_{e}\right),(2.8) \\
\operatorname{div} \tilde{u} & =0 .
\end{aligned}
$$

The terms in the braces on the right-hand side of eq. (2.8) represent the viscous dissipation acting on the large-scale eddies and the nonlinear dissipative effect of the small-scale eddies. These terms correspond to the terms on the right-hand side of eq. (1.5) expressed in the wavenumber space. Hence, if we employ the equipartition of dissipation (1.6), these terms are expressed as

$$
v \Delta \tilde{\mathrm{u}}-\langle(\hat{\mathrm{u}} \cdot \operatorname{grad}) \hat{\mathrm{u}}\rangle_{e}=-\gamma_{0}(t) \tilde{\mathrm{u}} \text {. }
$$

As stated in $\S 1$, the equipartition of dissipation (1.6) has a physical basis on the dynamical equilibrium of turbulence which is characterized by the self-similarity of statistical quantities. The equation (2.10), on the other hand, is an approximate relationship in the sense that the uniform dissipation is assumed for the velocity itself instead of the energy spectrum which depends only upon the magnitude of the velocity.

If we take the equi-dissipation relation (2.10) as the working hypothesis, the equation (2.8) for the large-scale eddies is written as

$$
\frac{\partial \tilde{u}}{\partial t}+(\tilde{u} \cdot \operatorname{grad}) \tilde{\mathrm{u}}=-\frac{1}{\rho} \operatorname{grad} \tilde{\mathrm{p}}-\gamma_{0} \tilde{\mathrm{u}} .
$$

The equation (2.11) together with eq. (2.9) and the relation (1.10) for $\gamma_{0}(t)$, provides us with a closed system of equations which governs the dynamics of the large-scale eddies of turbulence.

The equation for the turbulent energy contributed from the 
large-scale eddies is easily derived from eq. (2.11) as

$$
\frac{\mathrm{d}}{\mathrm{dt}}\left\langle\frac{1}{2}|\tilde{\mathrm{u}}|^{2}\right\rangle=-\gamma_{0}\left\langle|\tilde{u}|^{2}\right\rangle-\operatorname{grad}\left(\left\langle\frac{1}{2}|\tilde{\mathrm{u}}|^{2}+\frac{\tilde{\mathrm{p}}}{\rho}\right\rangle \tilde{\mathrm{u}}\right),
$$

where use has been made of eq.(2.9). For homogeneous turbulence, the last term on the right-hand side vanishes, so that eq. (2.12) is reduced to

$$
\frac{\mathrm{d}}{\mathrm{dt}}\left\langle\frac{1}{2}|\tilde{u}|^{2}\right\rangle+2 \gamma_{0}\left\langle\frac{1}{2}|\tilde{u}|^{2}\right\rangle=0
$$

In view of the fact that $\left\langle|\tilde{u}|^{2} / 2\right\rangle \approx\left\langle|u|^{2} / 2\right\rangle=\xi$, this equation is identical with the exact relation (1.9). This confirms the consistency of the approximation (2.10) with respect to the energetics of turbulence.

It may be interesting to note that the equation $(2.11)$ and (2.9) for the large-scale eddies can be written in an inviscid form. Under the transformation,

$$
\left.\begin{array}{l}
\tilde{u}(x, t)=\exp \left(-\int^{t} \gamma_{0}\left(t^{\prime}\right) d t^{\prime}\right) \tilde{U}(x, s), \\
\tilde{p}(x, t)=\exp \left(-2 \int^{t} \gamma_{0}\left(t^{\prime}\right) d t^{\prime}\right) \tilde{P}(x, s),
\end{array}\right\}
$$

and

$$
s=\int^{t} \exp \left(-\int^{t^{\prime}} \gamma_{0}\left(t^{\prime \prime}\right) d t^{\prime \prime}\right) d t^{\prime},
$$

eqs.(2.11) and (2.9) are written as

$$
\begin{aligned}
\frac{\partial \tilde{U}}{\partial s}+(\tilde{U} \cdot \operatorname{grad}) \tilde{U} & =-\frac{1}{\rho} \operatorname{grad} \tilde{P}, \\
\operatorname{div} \tilde{U} & =0,
\end{aligned}
$$

respectively, which are nothing but the Euler equations of motion for an inviscid fluid. An obvious consequence of this result is that the evolution of the large-scale eddies in turbulent flows is slowed down compared with that of the corresponding motion in an inviscid fluid since always $s<t$. 
Such a slowing-down effect of the large-scale eddies in turbulent flows may give a physical account for apparently very slow evolution of various huge-scale vortex structures which are observed in nature.

\section{Large-scale eddies in turbulent shear flows}

The treatment of the large-scale eddies described in $\$ 2$ can be extended to those in turbulent shear flows. The fluid velocity is now decomposed into the mean velocity $\bar{u}(x, t)$ and the two turbulent velocities $\tilde{\mathrm{u}}(\mathrm{x}, t)$ and $\hat{\mathrm{u}}(\mathrm{x}, t)$ introduced in $\S 2$,

$$
\begin{aligned}
& u(x, t)=\bar{u}(x, t)+\tilde{u}(x, t)+\hat{u}(x, t), \\
& \bar{u}(x, t)=\langle u(x, t)\rangle,\langle\tilde{u}(x, t)\rangle=\langle\hat{u}(x, t)\rangle=0,
\end{aligned}
$$

where the brackets denote the statistical average.

The equations for the mean motion are derived by substituting the decomposition (3.1) and the corresponding decomposition of the pressure,

$$
p(x, t)=\bar{p}(x, t)+\tilde{p}(x, t)+\hat{p}(x, t),
$$

into eqs. $(2.6)$ and $(2.7)$ as follows:

$$
\begin{aligned}
\frac{\partial \bar{u}}{\partial t}+(\bar{u} \cdot \operatorname{grad}) \bar{u} & =-\frac{1}{\rho} \operatorname{grad} \bar{p}+v \Delta \bar{u}-\langle(\tilde{u} \cdot \operatorname{grad}) \tilde{u}\rangle \\
& -\langle(\hat{u} \cdot \operatorname{grad}) \hat{u}\rangle, \\
\operatorname{div} \bar{u}= & 0,
\end{aligned}
$$

which are usually called as the Reynolds equations.

The equations for the turbulent velocities are obtained by subtracting eqs.(3.4) and (3.5) from the corresponding equations (2.6) and (2.7), Taking the average \langle\rangle$_{e}$ of the resultant equations, we obtain the following equations: 


$$
\begin{aligned}
\frac{\partial \tilde{u}}{\partial t}+ & (\bar{u} \cdot g r a d) \tilde{u}+(\tilde{u} \cdot \operatorname{grad}) \bar{u}+(\tilde{u} \cdot g r a d) \tilde{u}-\langle(\tilde{u} \cdot g r a d) \tilde{u}\rangle \\
& =-\frac{1}{\rho} \operatorname{grad} \tilde{p}+(\nu \Delta \tilde{u}-\langle(\hat{u} \cdot g r a d) \hat{u}\rangle=(\langle(\hat{u} \cdot g r a d) \hat{u}\rangle),
\end{aligned}
$$

$$
\operatorname{div} \tilde{u}=0 .
$$

According to the same spirit as the approximation (2.10), the terms in the braces on the right-hand side of eq. (3.6) is approximated as

$$
v \Delta \tilde{u}-\langle(\hat{u} \cdot g r a d) \hat{u}\rangle_{e}+\langle(\hat{u} \cdot g r a d) \hat{u}\rangle=-\gamma_{0} \tilde{u},
$$

where $\gamma_{0}$ is the turbulent dissipation rate defined by (1.10), but now it is generally dependent on the coordinates $x$. Under the approximation (3.8), the equation (3.6) is written as

$$
\begin{aligned}
& \frac{\partial \tilde{u}}{\partial t}+(\bar{u} \cdot g r a d) \tilde{u}+(\tilde{u} \cdot g r a d) \bar{u}+(\tilde{u} \cdot g r a d) \tilde{u}-\langle(\tilde{u} \cdot g r a d) \tilde{u}\rangle \\
& =-\frac{1}{\rho} \operatorname{grad} \tilde{\mathrm{p}}-\gamma_{0} \tilde{\mathrm{u}} \text {. }
\end{aligned}
$$

Thus, the equations (3.4), (3.5), (3.9) and (3.7) provide us with a closed set of equations for the mean velocity $\bar{u}(x, t)$ and the large-scale turbulent velocity $\tilde{u}(x, t)$, provided that the last term on the right-hand side of eq. (3.4) is ignored as a minor-order term.

The equation for the turbulent energy is derived from eq. (3.9) as follows:

$$
\begin{aligned}
& \frac{d}{d t}\left\langle\frac{1}{2}|\tilde{u}|^{2}\right\rangle+(\tilde{u} . g r a d)\left\langle\frac{1}{2}|\tilde{u}|^{2}\right\rangle+\frac{\partial \bar{u}_{i}}{\partial x_{j}}\left\langle\tilde{u}_{i} \tilde{u}_{j}\right\rangle \\
& =-2 \gamma_{0}\left\langle\frac{1}{2}|\tilde{u}|^{2}\right\rangle+\operatorname{grad}\left\langle\left(\frac{1}{2}|\tilde{u}|^{2}+\frac{\tilde{p}}{\rho}\right) \tilde{u}\right\rangle \\
& =-\varepsilon+\operatorname{grad}\left\langle\left(\frac{1}{2}|\tilde{u}|^{2}+\frac{\tilde{p}}{\rho}\right) \tilde{u}\right\rangle,
\end{aligned}
$$

where use has been made of eq.(1.10). The second and the third 
terms on the left-hand side of eq.(3.10) are usually called as the advection and the production terms respectively, while the gradient terms on the right-hand side are called as the diffusion terms. Comparison of eq.(3.10) with the exact equation derived from eq. (3.6) shows that the diffusion of the work done by the viscous dissipation is neglected in the present approximation compared with the diffusion due to the Reynolds stress and the pressure.

\section{Concluding remarks}

Recent progress in the flow visualization and the simulation of the flow field as a whole has made it possible to detect various ordered motions and determine their coherent structure hidden in apparently random fluctuations. Now it is generally recognized that turbulent flows involve large-scale eddies which have more or less coherent spatial structure. In turbulent flows bounded by solid walls, these coherent eddies are rather short-lived and soon transformed into random clusters of smaller eddies. In unbounded flows, on the other hand, they are more persistent and remain as clearly observable vortex motions such as the vortex row in a free shear layer and the Karmán vortex streets behind an obstacle in undoubtedly fully-developed turbulent flows. The statistical methods which are powerful in dealing with the statistical equilibrium state of small-scale components of turbulence are not adequate for describing such ordered motions, and any method of deterministic nature seems to be necessary for this purpose. The present formulation of the dynamics of 
the large-scale eddies in turbulent flows is hoped to be helpful for such a kind of works.

\section{References}

Orszag, S.A. (1977): Lectures on the statistical theory of turbulence. Balian, R. \& Peube, L. eds. Fluid Dynamics, Gordon and Breach, New York, pp.235-374.

Tatsumi, T. (1980): Theory of homogeneous turbulence. Adv. Appl. Mech. 20, pp. 39-133.

Tatsumi, T., Yamada, M. \& Takei, T. (1986): Factorized cumulant expansion for homogeneous turbulence. Fluid Dyn. Res. 1 , $59-75$. 